THE EUROPEAN BUSINESS ENVIRONMENT 


\title{
THE EUROPEAN BUSINESS ENVIRONMENT
}

\author{
Edited by
}

Neill Nugent and Rory O’Donnell 


\section{ISBN 978-0-333-56643-5 ISBN 978-1-349-23636-7 (eBook) \\ DOI 10.1007/978-1-349-23636-7}

Selection, editorial matter and Chapter 1 ( ) Neill Nugent and Rory O'Donnell 1994 Individual chapters (in order) $\odot$ Neill Nugent, Rory O'Donnell, Walter Cairns,

Martin Rhodes, Peter A. Vipond, John A. Murray and John Fahy, David Jacobson 1994 Softcover reprint of the hardcover 1st edition 1994 978-0-333-56642-8

All rights reserved. For information, write:

Scholarly and Reference Division,

St. Martin's Press, Inc., 175 Fifth Avenue,

New York, N.Y. 10010

First published in the United States of America in 1994

ISBN 978-0-312-12351-2

Library of Congress Cataloging-in-Publication Data

The European business environment / edited by Neill Nugent and Rory

O'Donnell.

p. $\mathrm{cm}$.

Includes bibliographical references (p. ) and index.

ISBN 978-0-312-12351-2

1. Europe-Commerce. 2. Europe-Economic policy. I. Nugent, Neill. II. O'Donnell, Rory.

HF3496.5.E945 1994

$338.94-\mathrm{dc} 20$ 


\section{Contents}

List of contributors vii

List of abbreviations and acronyms viii

Introduction Neill Nugent and Rory O'Donnell 1

1 The political environment $\quad 8$

Neill Nugent

2 The economic environment $\quad 47$ Rory O'Donnell

3 The legal environment $\quad 91$ Walter Cairns

4 Labour markets and industrial relations 121 Martin Rhodes

5 The financial environment 155 Peter A. Vipond

6 The marketing environment 183 John A. Murray and John Fahy

7 The technological and infrastructural environment 199 David Jacobson

$\begin{array}{ll}\text { References } & 237\end{array}$

Index $\quad 252$ 


\section{List of contributors}

Walter Cairns is Senior Lecturer in Law and Languages at Manchester Metropolitan University.

John Fahy is Lecturer in Strategic Marketing at Trinity College, Dublin.

David Jacobson is Senior Lecturer in Economics at Dublin City University.

John A. Murray is Professor of Business Studies at Trinity College, Dublin.

Neill Nugent is Professor of Politics at Manchester Metropolitan University.

Rory O'Donnell is Director of the National Economic and Social Council, Dublin.

Martin Rhodes is Lecturer in Government at Manchester University.

Peter A. Vipond is Principal Lecturer in Financial Services at London Guildhall University. 


\section{List of abbreviations and acronyms}

$\begin{array}{ll}\text { ACP } & \text { African, Caribbean and Pacific countries } \\ \text { BRITE } & \text { Basic Research in Industrial Technologies of Europe } \\ \text { BTG } & \text { British Technology Group } \\ \text { CAP } & \text { Common Agricultural Policy } \\ \text { CCP } & \text { Common Commercial Policy } \\ \text { CCITT } & \text { International Telephone and Telegraph Consultative Committee } \\ \text { CCT } & \text { Common Customs Tariff } \\ \text { CEA } & \text { European Insurance Committee } \\ \text { CEA } & \text { Energy Commission (France) } \\ \text { CEEP } & \text { European Centre of Public Enterprises } \\ \text { CEFIC } & \text { European Chemical Industry Federation } \\ \text { CEN } & \text { European Committee for Standardisation } \\ \text { CENELEC } & \text { European Committee for Electrotechnical Standardisation } \\ \text { CET } & \text { Common External Tariff } \\ \text { CNES } & \text { National Centre for Space Studies (France) } \\ \text { CNET } & \text { National Centre for the Study of Telecommunications (France) } \\ \text { CNRS } & \text { (French) National Centre for Scientific Research } \\ \text { COREPER } & \text { Committee of Permanent Representatives } \\ \text { DGA } & \text { Delegation Genérale a l'Armament } \\ \text { DSU } & \text { Deficit Spending Unit } \\ \text { EAGGF } & \text { European Agricultural Guidance and Guarantee Fund } \\ \text { EBRD } & \text { European Bank for Reconstruction and Development } \\ \text { EC } & \text { European Community } \\ \text { ECJ } & \text { European Court of Justice } \\ \text { ECU } & \text { European Currency Unit } \\ \text { EEA } & \text { European Economic Area } \\ \text { EEC } & \text { European Economic Community } \\ \text { EFA } & \text { European Financial Area } \\ \text { EFE } & \text { European Financial Environment } \\ \text { EFPIA } & \text { European Federation of Pharmaceutical Industry Associations } \\ \text { EFTA } & \text { European Free Trade Association } \\ \text { EIB } & \text { European Investment Bank } \\ \text { EMS } & \text { European Monetary System } \\ & \end{array}$




$\begin{array}{ll}\text { EMU } & \text { Economic and Monetary Union } \\ \text { EP } & \text { European Parliament } \\ \text { ERDF } & \text { European Regional Development Fund } \\ \text { ERM } & \text { Exchange Rate Mechanism } \\ \text { ESF } & \text { European Social Fund } \\ \text { ESPRT } & \text { European Strategic Programme for Research in Information } \\ & \text { Technology } \\ \text { ETUC } & \text { European Trade Union Confederation } \\ \text { EU } & \text { European Union } \\ \text { EUREKA } & \text { European Programme for High Technology Research and } \\ & \text { Development } \\ \text { FAST } & \text { Forecasting and Assessment in Science and Technology } \\ \text { FDI } & \text { Foreign Direct Investment } \\ \text { FRS } & \text { Financial Reporting Statement } \\ \text { GAAP } & \text { Generally Agreed Accounting Principles } \\ \text { GATT } & \text { General Agreement on Tariffs and Trade } \\ \text { GDP } & \text { Gross Domestic Product } \\ \text { GERD } & \text { Gross Domestic Expenditure on Research and Development } \\ \text { GNP } & \text { Gross National Product } \\ \text { GSM } & \text { Groupe Speciale Mobile } \\ \text { IAS } & \text { International Accounting Standards } \\ \text { IASC } & \text { International Accounting Standards Committee } \\ \text { ICT } & \text { Information and Communication Technologies } \\ \text { IEPG } & \text { Independent European Programme Group } \\ \text { IMF } & \text { International Monetary Fund } \\ \text { IOSCO } & \text { International Organisation of Securities Commission } \\ \text { ISDN } & \text { Integrated Services Digital Network } \\ \text { JV } & \text { Joint Venture } \\ \text { MAAs } & \text { Mergers, Acquisitions and Strategic Alliances } \\ \text { MEP } & \text { Member of the European Parliament } \\ \text { MFA } & \text { Multifibre Arrangement } \\ \text { MNC } & \text { Multinational Company } \\ \text { NAFTA } & \text { North American Free Trade Area } \\ \text { NATO } & \text { North Atlantic Treaty Organisation } \\ \text { NEB } & \text { National Enterprise Board (UK) } \\ \text { NSI } & \text { National System of Innovation } \\ \text { NTB } & \text { Non-Tariff Barrier } \\ \text { ONERA } & \text { National Office of Aeronautical Studies and Research (France) } \\ \text { OECD } & \text { Organisation for Economic Cooperation and Development } \\ \text { PTO } & \text { Public Telecommunications Operators } \\ \text { RACE } & \text { Research in Advanced Communications for Europe } \\ \text { R \& D } & \text { Research and Development } \\ \text { S \& T } & \text { Science and Technology } \\ \text { SEA } & \text { Single European Act } \\ \text { SEC } & \text { Securities and Exchange Commission } \\ & \end{array}$


$\mathrm{x} \quad$ List of abbreviations and acronyms

$\begin{array}{ll}\text { SEM } & \text { Single European Market } \\ \text { SME } & \text { Small and medium-sized Enterprise } \\ \text { SSAP } & \text { Statement of Standard Accounting Practice } \\ \text { SSU } & \text { Surplus Spending Unit } \\ \text { STAR } & \text { Special Telecommunications Actions for Regions } \\ \text { TBP } & \text { Technology Balance of Payments } \\ \text { TEU } & \text { Treaty on European Union } \\ \text { UK } & \text { United Kingdom } \\ \text { UNICE } & \text { Union of Industries of the European Community } \\ \text { USA } & \text { United States of America } \\ \text { VAT } & \text { Value Added Tax } \\ \text { VER } & \text { Voluntary Export Restraint }\end{array}$

